\title{
Assessment and Comparison of Cervical Column Morphology and Cranial Base Angle in Three Different Facial Types - A Cephalometric Study
}

\author{
Anshuka ${ }^{1}$, Varsha Vaswani ${ }^{2}$, Sahil Khajuria ${ }^{3}$ \\ 1Department of Orthodontics, VSPM Dental Collage and Reserch Center, Nagpur, Maharashtra, \\ India. ${ }^{2}$ Department of Orthodontics, VSPM Dental Collage and Reserch Center, Nagpur, \\ Maharashtra, India. ${ }^{3}$ Department of Surgery, GMC, Jammu and Kashmir, India.
}

\section{ABSTRACT}

\section{BACKGROUND}

We wanted to assess and compare the morphological deviations of the cervical vertebral column and cranial base angle in three different facial types that is hypodivergent, normodivergent and hyperdivergent groups and elucidate the aetiology behind the associations as well as clinical implications of the results.

\section{METHODS}

The study was conducted in the department of orthodontics wherein lateral cephalograms of 150 subjects in the age group of 18 - 25 years were taken and divided into three groups. The division of groups was made as per Steiner's mandibular plane angle i.e. Hyperdivergent group (SN-MP angle $>34$ degrees) Normodivergent group (SN-MP angle 26- 34 degrees) Hypodivergent group (SN-MP angle $<26$ degrees). Lateral cephalograms were traced and analysed to compare the variables by statistical analysis. Morphology of the cervical column was evaluated by visual inspection of the first 5 cervical vertebrae as they are normally seen on a standardized lateral skull radiograph. Characteristics of the cervical column were classified according to the method of Sandham. The cranial base angle was measured by the angle between nasion sella and sella basion (N-S-Ba) and then compared in the three groups.

\section{RESULTS}

The cranial base angle was increased significantly in the hyperdivergent group and decreased in the hypodivergent group when compared to the normodivergent group patients. However, no significant results were associated regarding the morphology of the cervical column in the three groups.

\section{CONCLUSIONS}

Associations between the cranial base angle and cervical column morphology plays a pivotal role in orthodontics in making the diagnosis of malocclusion and finding out the pattern of growth in vertical direction. A routine examination of the cervical vertebral column area for any deviation in the morphology should be done and checked. These registrations may prove useful in making the diagnosis and finding aetiology especially in the cases of severe skeletal malocclusions like severe open bite and deep bite and those with obstructive disorders like obstructive sleep apnoea or enlarged adenoids.

\section{KEY WORDS}

Cervical Column Morphology, Fusion Vertebrae, Occipitalization
Corresponding Author: Dr. Anshuka.

Otr/e9, Medical Enclave, Near KC Theatre, Bakshi Nagar, Jammu - 180001, Jammu and Kshmi, India. E-mail: anshukajasrotia@gmail.com

DOI: $10.14260 /$ jemds/2020/567

How to Cite This Article:

Anshuka, Vaswani V, Khajuria S. Assessment and comparison of cervical column morphology and cranial base angle in three different facial types- a cephalometric study. J Evolution Med Dent Sci 2020;9(36):2605-2609, DOI: $10.14260 /$ jemds/2020/567

Submission 01-06-2020,

Peer Review 25-07-2020,

Acceptance 03-08-2020,

Published 07-09-2020.

Copyright (C) 2020 JEMDS. This is an open access article distributed under Creative Commons Attribution License [Attribution 4.0 International (CC BY 4.0)] 


\section{BACKGROUND}

Deviations in the morphology of the cervical column have been found in healthy subjects with neutral occlusion and normal craniofacial morphology as well as in patients with craniofacial syndromes, severe malocclusion traits and deviating craniofacial morphology. ${ }^{1}$ Fusions between the upper cervical vertebrae (C2 and C3) have occurred in $14.3 \%$ of healthy subjects in a study. ${ }^{2}$ Thus the fusions of the upper cervical column within that range are considered normal. Studies have found an association between malformations of the upper cervical vertebrae and patients with cleft lip and palate. ${ }^{3-5} \mathrm{An}$ association was also found between malformation of the upper cervical vertebrae not only in patients with condylar hypoplasia, ${ }^{2}$ but also in adult orthodontic surgical patients with skeletal deep bite, ${ }^{6}$ skeletal mandibular overjet, ${ }^{7}$ skeletal horizontal overjet, and skeletal open bite. An association between the morphology of the cervical column and the posture of the head and neck has also been demonstrated by Sonnesen ${ }^{8}$ who in his study showed that fusion between C2 and C3 is associated with posture of the head and neck.

The posture of the head is influenced primarily by the force of gravity, but nevertheless the physiologic demands of the respiration, sight, balance, and hearing also must affect cranial deportment. Sollow and Tallgren and Posnick showed that statistical correlations exist between the predominant mode of respiration, head posture, and some facial features. Similarly, some studies showed that abnormal and prolonged changes in cranial posture during growth and maturation may have an effect on the expression of facial form. ${ }^{9}$ The developmental fault of the mesenchyme can be associated with the abnormal development of the cervical vertebrae and the mandible as these areas might be dependent on the same or similar para axial mesoderms ${ }^{3}$. As the morphology of the cervical column is associated with head posture and head posture is associated with craniofacial morphology, including the rotation pattern of the mandible, it could be hypothesized that there is an association between the morphology of the cervical column and the vertical dimensions of the face. ${ }^{9}$

Despite these observations, little is known about the relationship between the craniofacial morphology and craniofacial development related to different growth patterns. The aim of the present study is to assess and compare the morphological deviations of the cervical vertebral column and cranial base angle in three different facial types that is hypodivergent, normodivergent and hyperdivergent groups and to elucidate the aetiology behind the associations as well as clinical implications of the results.

\section{METHODS}

The study was conducted in the department of orthodontics wherein lateral cephalograms of 150 subjects in the age group of 18 - 25 years were taken and divided into three groups. The division of groups was made as per Steiner's mandibular plane angle i.e. Hyperdivergent group (SN-MP angle $>34$ degrees) Normodivergent group (SN-MP angle 26 - 34 degrees) Hypodivergent group (SN-MP angle $<26$ degrees).The subjects that were included in the study had no prior orthodontic treatment, no craniofacial anomalies or systemic muscle or joint disorders; all permanent teeth till second molars erupted and accessibility of a profile radiograph before orthodontic treatment with the first five cervical vertebral units visible. The subjects that were excluded from the study had a history of trauma to the cervical vertebrae or any previous surgery.

The statistical analysis was done by comparing the variables traced from the lateral cephalograms. The morphology of the cervical column was evaluated by visual inspection of the first 5 cervical vertebrae as they are normally seen on a standardized lateral skull radiograph. Characteristics of the cervical column were classified according to the method of Sandham ${ }^{5}$ and divided into 2 categories (Figure 1).

1. Posterior arch deficiency, defined as partial cleft and dehiscence. Partial cleft is defined as failure to fusion of the posterior part of the neural arch and dehiscence is defined as failure to development of a part of a vertebral unit.

2. Fusion anomalies, defined as fusion, block fusion, and occipitalization. Fusion is defined as fusion of 1 unit with another at the vertebral bodies, articulation facets, neural arch, or transverse processes. Occipitalization is defined as assimilation, either partially or completely, of the atlas (C1) with the occipital bone. Block fusion is defined as fusion of more than 2 units at the vertebral bodies, articulation facets, neural arch, or transverse processes.

Only anomalies verified on the lateral profile radiographs were registered as anomalies of the cervical vertebral column.

For the craniofacial dimensions, the profile radiographs were taken with the teeth in occlusion and in the standardized natural head posture, the mirror position, as described by Siersbaek-Nielsen and Solow ${ }^{10}$. The cranial base angle was measured by the angle between nasion sella and sella basion (N-S-Ba).

\section{Reliability}

Reliability of the visual assessment of the morphologic characteristics of the cervical vertebral units was determined by interobserver examination, which showed very good agreement (0.82) as assessed by the kappa coefficient. The reliability of the angle representing cranial base was done by selecting 20 lateral cephalograms randomly and then tracing them manually again. The measurements obtained were checked with the previous recordings and paired t tests found no significant differences between the 2 sets of recordings.

\section{Statistical Analysis}

Statistical comparison of morphology patterns across groups was performed using Pearson's Chi - square test. The analyses were performed in SPSS ver. 20.0 (IBM Inc.) and the significance was tested at $5 \%$ level

\section{RESULTS}

Table I provides the frequency distribution of morphology in the three groups. In hyperdivergent group, 14 (46.7 \%) cases 
had fusion C2 - C3 morphology, while 13 (43.4 \%) had normal and only $3(10 \%)$ had block fusion morphology. In the normodivergent group, 17 (56.7 \%) had normal, 9 (30.0\%) had fusion C2 - C3 and 4 (13.3\%) had block fusion morphology. In the hypodivergent group, 13 (43.3) cases each had normal and fusion C2 - C3 morphology, while 4 (13.3\%) had block fusion morphology.

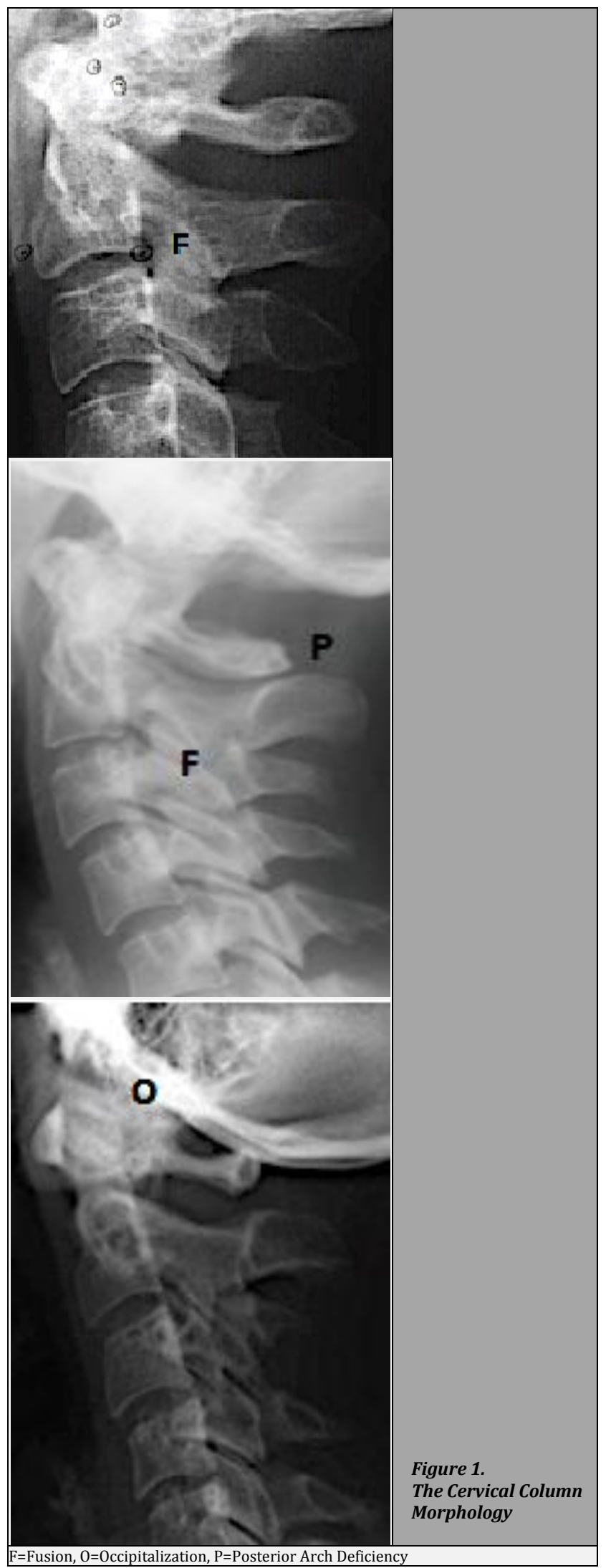

\begin{tabular}{|ccccc|}
\hline Groups & Normal & $\begin{array}{c}\text { No. ( \% ) } \\
\text { C2-C3 }\end{array}$ & $\begin{array}{c}\text { Block } \\
\text { Fusion }\end{array}$ & P - Value* \\
Hyperdivergent $(\mathrm{n}=30)$ & $13(43.3)$ & $14(46.7)$ & $3(10.0)$ & \\
Normodivergent $(\mathrm{n}=30)$ & $17(56.7)$ & $9(30.0)$ & $4(13.3)$ & $0.718(\mathrm{NS})$ \\
Hypodivergent (n=30) & $13(43.3)$ & $13(43.3)$ & $4(13.3)$ & \\
\hline \multicolumn{4}{|c|}{ Table I. Frequency Distribution of Morphology in the Three Groups } \\
\hline *obtained using Chi-square test; NS: Non-Significant \\
\hline
\end{tabular}

The association of groups and morphology pattern was studied for statistical significance using Chi-square test, which resulted into a P-value of 0.718 , suggesting that there is no association between two factors.

Table II provides the descriptive statistics for cranial base angle measurement for three groups. The mean N-S-Ba for hyperdivergent group was 126.28 (4.26), while for normodivergent group was 124.30 (5.03) and hypodivergent group was 122.48 (4.76). The difference in the means was statistically significant with $\mathrm{P}$-value of $0.03(\mathrm{P}<0.05)$ using one-way ANOVA. Thus, an increased cranial base angle was seen in hyperdivergent patients and vice versa in hypodivergent patients.

\begin{tabular}{|cccccc|}
\hline Parameters & Groups & Mean & SD & SE & P-Value* \\
& Hyperdivergent & 126.28 & 4.26 & 1.56 & \\
N-S-Ba & Normodivergent & 124.30 & 5.03 & 1.84 & 0.03 \\
& Hypodivergent & 122.48 & 4.76 & 1.74 & \\
\hline \multicolumn{4}{|c|}{ Table II. Descriptive Statistics for Cranial Base Angle } \\
& in the Three Groups \\
\hline *Obtained using ANOVA test & \\
\hline
\end{tabular}

\section{DISCUSSION}

Cervical column morphology is examined by checking for any posterior arch deficiency, fusion anomalies, block fusion, and occipitalization. The literature has shown a strong association of the presence of deviation in the normal morphology of the cervical vertebral column and the vertical growth pattern. Fusion anomalies and block fusions have been found in open bite as well as deep bite cases which are associated with hyperdivergent and hypodivergent growth respectively. However, in the present study the association of groups and morphology pattern was not statistically significant in three groups with a p-value of 0.718 , suggesting that there is no association between two factors. These results were not expected, since a previous study has shown that there was a significant deviation in the morphology of the cervical column in a group of children with mandibular retrusion compared with children having proclined maxillary teeth. ${ }^{11}$ Another study by Sonneson ${ }^{2}$ (2007) wherein the group having skeletal deep bite when compared to the one with normal occlusion showed significant deviation in the morphology of the cervical column.

Furthermore, significant correlation in the deviation of cervical column morphology has been found in patients with severe skeletal malocclusions when compared with the patients having normal occlusion as well as craniofacial morphology.

The possible explanation for non-significant results in our study could be that the vertical craniofacial deviations in the hypodivergent and hyperdivergent groups were not that severe. The vertical jaw relationship in hyperdivergent group ranged from $35^{\circ}-42^{\circ}$ and in hypodivergent group ranged from 
$11^{\circ}-24^{\circ}$. However the groups of adult surgical patients with severe skeletal malocclusions included in above mentioned study had the vertical jaw relationships of more than $40^{\circ}$.

The false positive results on the lateral cephalograms due to pseudo -fusions or change in the orientation of cervical column and superimposition of the cervical vertebrae can also be responsible for the insignificant results in the present study. The risk of the false positive readings was minimized by assuming the morphologic deviations to be normal for that particular cephalogram in case of any doubt.

However, we found one study in literature (Kim and Sonneson ${ }^{12} 2014$ ) that showed results similar to our study. In their study no significant differences in the cervical column's morphologic deviations were found between the skeletal and dentoalveolar open-bite groups. The reasons for these deviations in the cervical column with different prevalence is still unclear. Further, the different patterns of the skeletal craniofacial morphology and malocclusion traits cannot be explained. However, it is possible that in the early prenatal period, the notochord might play a role in localizing and shaping the vertebral bodies (Arntsen and Sonneson ${ }^{13}$, 2011).

A previous study on preorthodontic surgical adult patients showed a clear association between morphologic deviations of the cervical vertebral column and a large cranial base angle, retrognathia of the jaws, and increased inclination of the jaws. Another study by Sonneson ${ }^{6}$ (2007) showed association between the morphological deviations of the upper cervical vertebrae and deep bite as well as vertical craniofacial morphology.

The period of early embryogenesis can establish an association between the deviation of cervical column morphology and craniofacial morphology including the cranial base. As both the cervical vertebral development and occipital bone formation is dependent on the notochord, a relation can be established between the cranial base angle and the deviation in cervical vertebral morphology ${ }^{1}$. Therefore, it can be suggested that the cranial base could be a developing link between the cervical vertebral column and jaws. Bjork in his study found an association between the cranial base angle and the craniofacial morphology. It was found that the subjects having large cranial base angle in adulthood were having retrognathic jaws and also an increase in the vertical angle of the jaws. Furthermore, a genetic interrelationship between the body axis and the basilar part of the occipital bone and between the body axis which surrounds the notochord and the craniofacial region have been shown by the immunehistochemical studies on wild-type mouse embryos. ${ }^{1}$

In the present study contradictory results were found with the results mentioned above. No associations were established between the deviations in the cervical column morphology and the craniofacial dimension but significant differences were found in the head posture and craniofacial dimensions in different groups. This could indicate that the etiological factors contributing to varying growth patterns and varied head postures in different groups could be attributed to other environmental factors like respiratory obstruction, tongue posture in hyperdivergent patients than an embryologic deviation in the early development, as a signalling of the notochord of which vertebral morphologic deviations could be a sign.

The cranial base angle is usually measured radiographically as the angle between the basion-sella-nasion points. The angle at birth is approximately $142^{\circ}$, but then reduces to $130^{\circ}$ at 5 years of age and from 5 to 15 years this angle remains stable. However, the change in the cranial base angle can be determined early in life with the change in the growth pattern in vertical direction. As the cranial base angle depends on the position of glenoid fossa. Any change in the position of the fossa would redirect the direction in which the mandible grows. Hence the cranial base angle is influenced by the hyperdivergent and hypodivergent growth patterns.

Bjork $^{14}$ (1983) in his study showed an association of the cranial base angle and the sagittal dimension of the jaws. An acute angle was observed when the mandible was placed forwardly and an obtuse angle was seen with a retruded mandible. Similar results were shown by DeCoster $\mathrm{H}$ and Sassouni ${ }^{15}$ who suggested an association between a large cranial base angle and open bite and, conversely, between smaller cranial base angles and deep bite. Similarly, in this study an increased cranial base angle was found in hyperdivergent subjects and decreased cranial base angle was seen in hypodivergent subjects when compared to the normodivergent patients.

\section{CONCLUSIONS}

In hyperdivergent group, $46.7 \%$ cases had fusion C2 - C3 morphology and $10 \%$ had block fusion morphology. In the normodivergent group, $30.0 \%$ had fusion C2 - C3 and $13.3 \%$ had block fusion morphology. In the hypodivergent group, $43.3 \%$ cases had fusion C2 - C3 morphology, while $13.3 \%$ had block fusion morphology. The association of groups and morphology patterns was studied for statistical significance using Chi-square test, which resulted in a P-value of 0.718 , suggesting that there is no association between two factors.

An increased cranial base angle was found in hyperdivergent subjects and decreased cranial base angle was seen in hypodivergent subjects when compared to the normodivergent patient.

Cases of severe skeletal malocclusion such as severe open bite cases and cases of obstructive sleep apnoea had an increase in the cranial base angle while those of severe deep bite cases showed a significantly reduced cranial base angle when compared to the normal groups. Thus, a routine examination of the cervical vertebral column area for any deviation in the morphology should be done and checked. These registrations may prove useful in making the diagnosis and finding the aetiology.

Financial or Other Competing Interests: None.

\section{REFERENCES}

[1] Kim P, Sarauw MT, Sonnesen L. Cervical vertebral column morphology and head posture in preorthodontic patients with anterior open bite. Am J Orthod Dentofacial Orthop 2014;145(3):359-66.

[2] Sonnesen L, Pedersen C, Kjaer I. Cervical column morphology related to head posture, cranial base angle and condylar malformation. Eur J Orthod 2007;29(4):398-403. 
[3] Sandham A. Cervical vertebral anomalies in cleft lip and palate. Cleft Palate J 1986;23(3):206-14.

[4] Horswell BB. The incidence and relationship of cervical spine anomalies in patients with cleft lip and/or palate. J Oral Maxillofac Surg 1991;49(7):693-7.

[5] Ugar DA, Semb G. The prevalence of anomalies of the upper cervical vertebrae in subjects with cleft lip, cleft palate, or both. Cleft Palate Craniofac J 2001;38(5):498503.

[6] Sonnesen L, Kjaer I. Cervical vertebral body fusions in patients with skeletal deep bite. Eur J Orthod 2007;29(5):464-70.

[7] Sonnesen L, Kjaer I. Cervical column morphology in patients with skeletal Class III malocclusion and mandibular overjet. Am J Orthod Dentofacial Orthop 2007;132(4):427.e7-12.

[8] Sonnesen L. Associations between the cervical vertebral column and craniofacial morphology. Int J Dent 2010;2010:1-6.

[9] Solow B, Tallgren A. Head posture and craniofacial morphology. Am J Phys Anthropol 1976;44(3):417-35.
[10] Siersbaek-Nielsen S, Solow B. Intra- and interexaminer variability in head posture recorded by dental auxiliaries. Am J Orthod 1982;82(1):50-7.

[11] Kjaer I. Human prenatal craniofacial development related to brain development under normal and pathologic conditions. Acta Odontologica Scandinavica 1995;53(3):135-44.

[12] Kim P, Sarauw MT, Sonnesen L. Cervical vertebral column morphology and head posture in preorthodontic patients with anterior open bite. Am J Orthod Dentofacial Orthop 2014;145(3):359-66.

[13] Arntsen T, Sonnesen L. Cervical vertebral column morphology related to craniofacial morphology and head posture in preorthodontic children with Class II malocclusion and horizontal maxillary overjet. Am J Orthod Dentofacial Orthop 2011;140:e1-7.

[14] Bjork A, Skieller V. Normal and abnormal growth of the mandible. A synthesis of longitudinal cephalometrie implant studies over a period of 25 years. Eur J Orthod 1983;5(1):1-46.

[15] DeCoster L. Open-bite. Int J Orthod Oral Surg 1936;22:912-38. 